\title{
Rate of tuberculosis infection in children and adolescents with household contact with adults with active pulmonary tuberculosis as assessed by tuberculin skin test and interferon-gamma release assays
}

\author{
M. A. G. FERRARINI ${ }^{1}$, F. G. SPINA ${ }^{1}$, L. Y. WECKX ${ }^{1}$, H. M. LEDERMAN ${ }^{2}$ AND \\ M. I. DE MORAES-PINTO ${ }^{1 *}$ \\ ${ }^{1}$ Department of Pediatrics, Universidade Federal de São Paulo, São Paulo, SP, Brazil \\ ${ }^{2}$ Departament of Diagnostic Imaging, Universidade Federal de São Paulo, São Paulo, SP, Brazil
}

Received 30 December 2014; Final revision 9 June 2015; Accepted 7 July 2015;

first published online 3 August 2015

\section{SUMMARY}

Tuberculosis (TB) infection was evaluated in Brazilian immunocompetent children and adolescents exposed and unexposed (control group) to adults with active pulmonary TB. Both groups were analysed by clinical and radiological assessment, TST, QFT-IT and T-SPOT.TB. The three tests were repeated after 8 weeks in the TB-exposed group if results were initially negative. Individuals with latent tuberculosis infection (LTBI) were treated and tests were repeated after treatment. Fifty-nine TB-exposed and 42 controls were evaluated. Rate of infection was $69 \cdot 5 \%$ and $9 \cdot 5 \%$ for the exposed and control groups, respectively. The exposed group infection rate was $61 \%$ assessed by TST, $57 \cdot 6 \%$ by T-SPOT.TB, and $59 \cdot 3 \%$, by QFT-IT. No active TB was diagnosed. Agreement between the three tests was $83 \cdot 1 \%$ and $92 \cdot 8 \%$ in the exposed and control groups, respectively. In the exposed group, T-SPOT.TB added four TB diagnoses [16\%, 95\% confidence interval (CI) 1.6-30.4] and QFT-IT added three TB diagnoses $(12 \%, 95 \%$ CI $0-24 \cdot 7)$ in 25 individuals with negative tuberculin skin test (TST). Risk factors associated to TB infection were contact with an adult with active TB [0-60 days: odds ratio (OR) 6.9; >60 days: OR 27.0] and sleeping in the same room as an adult with active TB (OR 5.2). In Brazilian immunocompetent children and adolescents, TST had a similar performance to interferon-gamma release assays and detected a high rate of LTBI.

Key words: Adolescents, BCG, children, IGRA, latent tuberculosis infection, TST.

\section{INTRODUCTION}

Approximately 2 billion people worldwide have latent Mycobacterium tuberculosis infection (LTBI) and are at risk of progression to tuberculosis (TB) disease. Young children are at increased risk of developing serious disease due to decreased ability of their immune system to contain infection [1]. An essential strategy

\footnotetext{
* Author for correspondence: M. I. de Moraes Pinto, MD, PhD, Rua Pedro de Toledo, 781/9 ${ }^{\circ}$ andar, São Paulo, SP 04039-032, Brazil.

(Email: m.isabelmp@uol.com.br)
}

to eliminate TB is treatment of LTBI in order to avoid illness in infected target groups [2,3].

Tuberculin skin test (TST) has been used as the sole immunodiagnostic test for the detection of Mycobacterium infection. However, it has limitations such as low specificity due to cross-reaction with nontuberculous mycobacteria (NTM) and Mycobacterium bovis bacillus Calmette-Guérin (BCG) vaccine strains. Moreover, low sensitivity is described in young children and immunosuppressed individuals [2, 4].

At present two commercial interferon-gamma release assays (IGRAs): T-SPOT.TB (Oxford 
Immunotec, UK) and QuantiFERON-TB Gold In-Tube assay (QFT-IT; Cellestis, Australia) based on the detection interferon-gamma (IFN- $\gamma$ ) by $\mathrm{T}$ cells after in vitro exposure to antigens from $M$. tuberculosis are available.

Although IGRAs are expensive and require an equipped laboratory with trained staff, they have operational advantages over TST, and they appear to have higher specificity compared to TST. However, like TST, they cannot distinguish between latent and active TB disease [4].

Most early studies comparing TST and IGRA were performed in adults [5] or in mixed samples of adults and children [6,7], but few studies were performed exclusively in children $[8,9]$. More recently, other studies have been conducted exclusively in children [10-14].

To the best of our knowledge, no studies comparing TST and IGRA in children only who were household contacts of adults with active pulmonary TB have been performed in Brazil.

The goals of this study were:

(1) To describe the total rate of $M$. tuberculosis infection in children and adolescents with active TB household contact across three tests: TST, T-SPOT.TB and QFT-IT and to compare this rate in children and adolescents without known TB exposure.

(2) To describe the LTBI rate for each test in the same groups of children and adolescents.

(3) To describe the diagnostic increment rate with TST repetition, as recommended by the Ministry of Health in Brazil when the TST is negative at the first evaluation and the diagnostic increment rate when each IGRA is performed in parallel to TST.

\section{METHODS}

This is a prospective study performed at the Federal University of São Paulo, Brazil, from August 2010 to June 2013. Two groups of children and adolescents aged $<15$ years were investigated: one group with household contact with adults with active TB (known exposure group) and another group without known contact with TB (control group).

The control group was composed of children and adolescents without known contact with $\mathrm{TB}$ and who lived with healthy adults without respiratory symptoms and who had never been investigated or treated for TB. They were age- and gender-matched to the children and adolescents from the known exposure group.

Adult cases of active $\mathrm{TB}$ had sputum and/or bronchoalveolar lavage (BAL) positive smear or a positive culture for $M$. tuberculosis complex. The exposure period was defined as the period of time in days from the onset of clinical symptoms of the index case until study entry.

Children and adolescents in both groups with previous TB treatment or with known primary or secondary immunodeficiency or a clinical condition suggestive of possible immunodeficiency were excluded.

Individuals from known exposure and control groups were submitted to a clinical evaluation performed by one of the authors (M.A.G.F.). They were then sent for chest radiography and tested for TST, T-SPOT.TB, QFT-IT and HIV infection.

Those from the control group were discharged after the first evaluation if TST and the two IGRAs proved negative, and the chest radiography did not present any image suggestive of pulmonary disease.

Children and adolescents from the known exposure group had another set of tests performed after 8 weeks if they were all negative at the first evaluation. When one of the three tests was positive either at the first evaluation or after 8 weeks and there was no evidence of disease, treatment for LTBI was initiated. They were then seen every 30 days by the same paediatrician (M.A.G.F.) for 6 months, after which laboratory re-evaluation was performed (Fig. 1).

The study protocol was approved by the Ethics Committee of the Federal University of São Paulo, Brazil (protocol no. 2121/08). All parents or guardians of eligible participants gave written informed consent for study participation before enrolment.

\section{Sample size assessment}

Because no previous study of this kind had been performed in the Brazilian population, the expected percentage of discordance between the three tests (TST and the two IGRAs) was arbitrarily set at $10 \%$ for the control group and 15\% for the known exposure group.

A sample size of 39 individuals without known contact with TB was necessary in order to obtain an estimate of percentage of discordance between the three tests that did not differ from the true value by more than $30 \%$ (margin of relative error), with a $95 \%$ confidence interval (CI). For the group of individuals with known contact with TB, a sample size of 53 was necessary in order to obtain an estimate of percentage of 


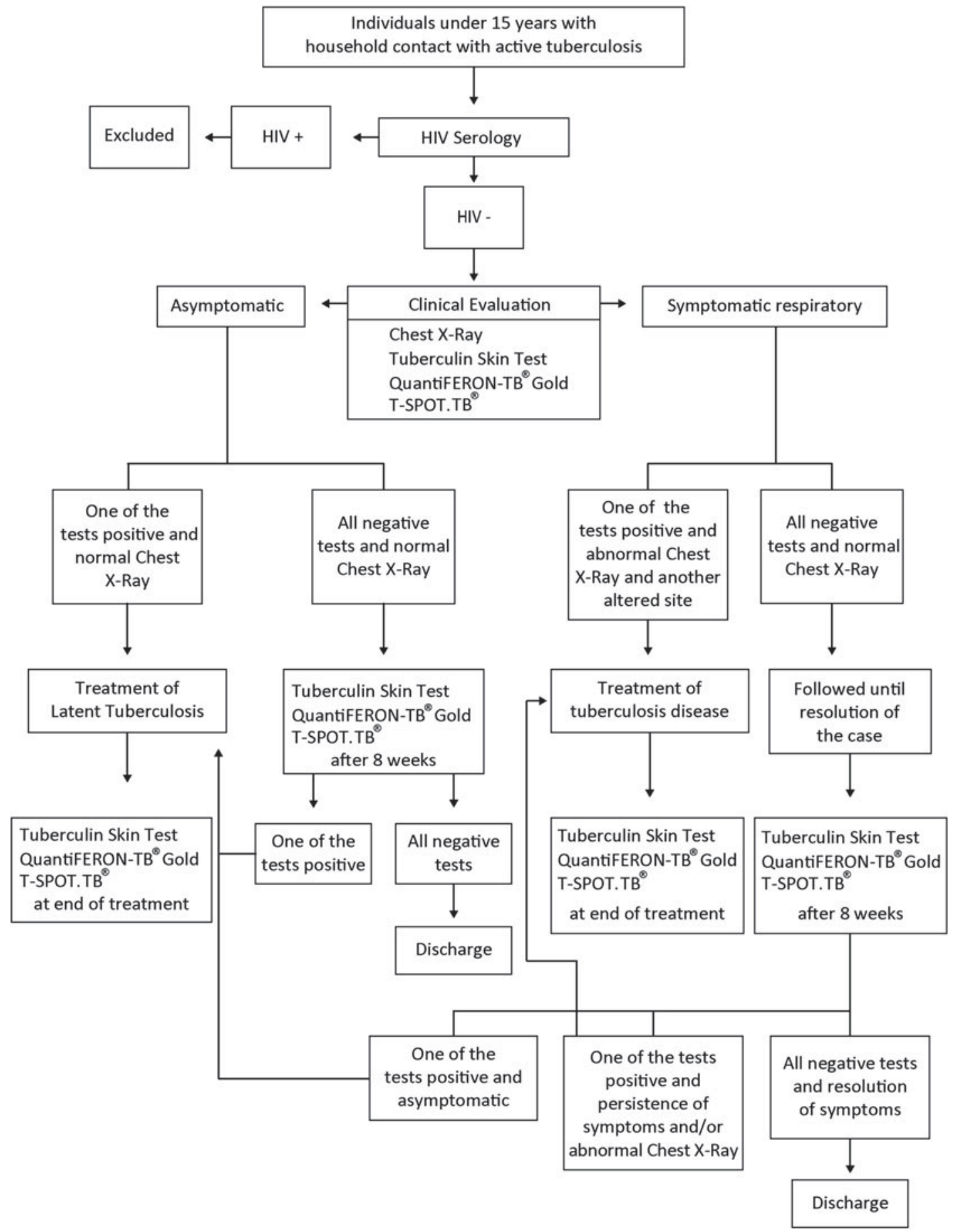

Fig. 1. Algorithm of the known exposure group of children and adolescents.

discordance between the three tests that did not differ from the true value by more than $25 \%$ (margin of relative error), with a $95 \% \mathrm{CI}$.

\section{Nutritional evaluation}

Participants from both groups were evaluated for $Z$ score body mass index using the World Health
Organization reference values for children aged $<5$ years [15] and for those aged 5-19 years [16].

\section{Socioeconomic classification}

The socioeconomic classification of each adult case was assessed using a questionnaire from the Brazilian Association of Research Companies [17], which 
estimates the purchasing power of individuals and families according to the possession of items and the level of education of the head of the family. The final score ranks economic class from $\mathrm{A}$ (the highest) to $\mathrm{E}$ (the lowest).

\section{TST}

Two units of purified protein derivative (PPD) RT23 (Statens Serum Institute, Denmark) were applied to the volar surface of the left lower forearm according to the intradermal Mantoux method. A trained nurse assessed the transverse diameter of skin induration in millimetres after $72 \mathrm{~h}$ of PPD administration. TST results were considered positive depending on previous BCG vaccination: $\geqslant 5 \mathrm{~mm}$, when the child had received BCG more than 2 years previously and $\geqslant 10 \mathrm{~mm}$, when BCG was administered in the last 2 years [18].

\section{Laboratory assays for TB immunodiagnostic}

Peripheral blood samples were obtained simultaneously for both assays and all blood samples were processed within $2 \mathrm{~h}$ of phlebotomy, as recommended. The commercially available T-SPOT.TB (Oxford Immunotec, UK) and the QuantiFERON-TB Gold In-tube (QFT-IT) system (Cellestis, Australia) were used in all assays and performed according to the manufacturers' instructions.

\section{HIV testing}

All children and adolescents were submitted to a HIV serology test. The Determine ${ }^{\mathrm{TM}}$ HIV-1/2 kit (Abbott, Japan) was used following the manufacturer's recommendations.

\section{Chest radiography}

All children were submitted to chest radiography at the first evaluation. All chest radiographies were analysed by a single experienced staff radiologist (H.M.L.), who was blind to the clinical data.

\section{Definitions and treatment}

The criteria established by III Brazilian Thoracic Association on Tuberculosis (2009) [18] were employed.

\section{LTBI}

LTBI was considered in children and adolescents aged $<15$ years who presented a positive TST with no signs of active disease. Alternatively, for this study, children or adolescents with positive results for QFT-IT or T-SPOT.TB were also considered infected.

LTBI treatment consisted of isoniazid at a dose of $5-10 \mathrm{mg} / \mathrm{kg}$ per day (maximum $300 \mathrm{mg} /$ day) for 6 months [18].

\section{Active pulmonary $T B$}

Diagnosis of active pulmonary TB was considered according to a scoring system recommended by the Brazilian National Ministry of Health which was based on clinical and radiological findings, known contact with an adult with TB in the last 2 years, TST result and nutritional status [19].

\section{Statistical analysis}

Categorical variables were compared using $\chi^{2}$ test or Fisher's exact test. Continuous variables with nonnormal distributions were compared with the nonparametric Mann-Whitney test. Agreement between tests was evaluated using the Kappa test. McNemar's test was employed to compare qualitative response change of QFT-IT and T-SPOT.TB after treatment. The paired $t$ test or Wilcoxon's test were used to assess quantitative alterations of TST and T-SPOT.TB after treatment.

Univariate and multivariate logistic regression analyses were used to evaluate the association between selected demographic and patients' characteristics and LTBI diagnosis. The following risk factors for TB infection were analysed: gender, age, period of exposure to index case and sleeping in the same room as index case.

A $P$ value $<0.05$ was considered statistically significant. All analyses were performed using Minitab ${ }^{\circledR}$ 16-1 (Minitab, USA) and SPSS statistics v. 20.0 (IBM, USA).

\section{RESULTS}

Of the initial sample of 111 children and adolescents, 10 were excluded (six from the known exposure group and four from the control group), so that 101 children and adolescents (59 from known exposure group and 42 from control group) were included in the study. 
Table 1. Characteristics of children and adolescents from the known exposure group and control group

\begin{tabular}{|c|c|c|c|}
\hline Parameter & Known exposure group $(n=59)$ & Control group $(n=42)$ & $P$ value \\
\hline Median age (years) (Q1-Q3) & $5 \cdot 3(3 \cdot 2-10 \cdot 5)$ & $7 \cdot 3(5 \cdot 4-10 \cdot 5)$ & $0 \cdot 167 \dagger$ \\
\hline Female gender $(\%)$ & $31 / 59(52 \cdot 5 \%)$ & $24 / 42(57 \cdot 1 \%)$ & $0 \cdot 799 \dagger$ \\
\hline Relationship & $\begin{array}{l}\text { Daughter/son: 22/59 }(37 \cdot 3 \%) \\
\text { Grandchild: } 12 / 59(20 \cdot 3 \%) \\
\text { Niece/nephew: } 15 / 59(25 \cdot 4 \%) \\
\text { Stepson: } 2 / 59(3 \cdot 4 \%) \\
\text { Brother: } 2 / 59(3 \cdot 4 \%) \\
\text { Cousin: } 3 / 59(5 \cdot 1 \%) \\
\text { Foster care contact: } 3 / 59(5 \cdot 1 \%)\end{array}$ & $\begin{array}{l}\text { Daughter/son: } 38 / 42 \\
(90 \cdot 5 \%) \\
\text { Grandchild: } 1 / 42(2 \cdot 4 \%) \\
\text { Niece/nephew: } 3 / 42(7 \cdot 0 \%)\end{array}$ & n.a. \\
\hline Exposure period & $\begin{array}{l}\leqslant 30 \text { days: } 15 / 59(25 \cdot 4 \%) \\
31-60 \text { days: } 15 / 59(25 \cdot 4 \%) \\
61-90 \text { days: } 14 / 59(23 \cdot 7 \%) \\
\geqslant 91 \text { days: } 15 / 59(25 \cdot 4 \%)\end{array}$ & $0 / 42(0 \%)$ & n.a. \\
\hline Nutritional assessment & $\begin{array}{l}\text { Eutrophic: } 44 / 59(74 \cdot 6 \%) \\
\text { Overweight risk: } 4 / 59(6 \cdot 8 \%) \\
\text { Overweight: } 5 / 59(8 \cdot 5 \%) \\
\text { Obesity: } 2 / 59(3 \cdot 4 \%) \\
\text { Slimness: } 4 / 59(6 \cdot 8 \%)\end{array}$ & $\begin{array}{l}\text { Eutrophic: } 29 / 42(69 \cdot 0 \%) \\
\text { Overweight risk: } 2 / 42 \\
(4 \cdot 8 \%) \\
\text { Overweight: } 6 / 42(14 \cdot 3 \%) \\
\text { Obesity: } 5 / 42(11 \cdot 9 \%)\end{array}$ & $0.933 \$$ \\
\hline Relevant clinical findings & $\begin{array}{l}\text { Pneumonia responsive to antibiotics: } 2 / 59 \\
(3 \cdot 4 \%)\end{array}$ & $0 / 42(0 \%)$ & n.a. \\
\hline $\begin{array}{l}\text { Altered chest radiography on } \\
\text { admission }\end{array}$ & $1 / 59(1 \cdot 7 \%)^{*}$ & $0 / 42(0 \%)$ & n.a. \\
\hline
\end{tabular}

n.a., Not applicable.

* Interpreted as primary complex, with resolution in the following radiography.

$\dagger$ Mann-Whitney test.

$+\chi^{2}$ test.

Individuals from both groups were comparable with regard to age, gender and nutritional status (Table 1). Median BCG vaccination age was comparable between groups (exposure group 19 days vs. control group 15 days; Mann-Whitney, $P=0 \cdot 361$ ). Only one child from the exposure group did not have a BCG scar or records to prove their BCG vaccination. The largest number of index cases in the contact group were parents $(22 / 59,37 \cdot 3 \%)$ and they belonged to socioeconomic group C $(60 \cdot 6 \%)$ and group B (30.3\%) (Tables 1 and 2).

Of the 59 children and adolescents from the known exposure group, $38(64 \cdot 4 \%)$ were submitted to treatment for LTBI due to positivity of one or more tests in the first assessment. Twenty-one $(21 / 59,35 \cdot 6 \%)$ had negative results and were invited to repeat the three tests, but two refused, therefore 19 children repeated the tests after 8 weeks. Of these, three were diagnosed with LTBI and commenced treatment.

Of the 42 children and adolescents from the control group, 38 presented negative results in all the tests at the first evaluation and were then discharged. Four $(4 / 42,9 \cdot 5 \%)$ presented one or more positive tests and commenced treatment for LTBI and were retested after finishing. Of those four controls, only one child had positive results in the three tests. Two children were positive only for TST and one child was positive for QFT-IT and negative for TST and T-SPOT.TB.

No cases of children or adolescents with active TB were diagnosed.

Of 45 LTBI children who started treatment, three abandoned the follow-up, one in the second month and two in the fifth month (Table 3).

The agreement between the three tests was $83 \cdot 1 \%$ (49/59) for the known exposure group at the first evaluation and $92 \cdot 8 \%$ (39/42) for the control group (Kappa TST vs. QFT-IT $=0 \cdot 807$, TST vs. T-SPOT. $\mathrm{TB}=0.798$ and QFT-IT $v s . \quad$ T-SPOT.TB $=0.888$ ) (Fig. 2).

In the known exposure group, TST was positive in $36 / 59$ children (61\%), 34 at the first evaluation and two in the second series of tests, with an increment of $10 \cdot 5 \%(95 \%$ CI $0 \cdot 0-24 \cdot 3)$.

With respect to IGRAs, two indeterminate results for the T-SPOT.TB at the first evaluation were excluded from the analyses. 
Table 2. Demographic data of adult index cases with active tuberculosis

\begin{tabular}{ll}
\hline \hline Parameter & Description \\
\hline Median age (years) (Q1-Q3) & $34(26-40)$ \\
Female gender (\%) & $21 / 33(64 \cdot 0 \%)$ \\
Socioeconomic classification (\%) & A: $2 / 33(6 \cdot 1 \%)$ \\
& B: $10 / 33(30 \cdot 3 \%)$ \\
& C: $20 / 33(60 \cdot 6 \%)$ \\
Number of contacts (\%) & D: $1 / 33(3 \cdot 0 \%)$ \\
& One: $13 / 33(39 \cdot 4 \%)$ \\
AFB in sputum (\%) & Two: $15 / 33(45 \cdot 5 \%)$ \\
& Three to five: $5 / 33(15 \cdot 1 \%)$ \\
& Negative: $3 / 33(9 \cdot 1 \%)^{*}$ \\
Sputum culture for Mycobacterium tuberculosis complex $(\%)$ & $+: 8 / 33(24 \cdot 2 \%)$ \\
& $++: 4 / 33(12 \cdot 1 \%)$ \\
& $+++: 6 / 33(18 \cdot 2 \%)$ \\
& Positive without quantification: $12 / 33(36 \cdot 4 \%)$ \\
& Done: $16 / 33(48 \cdot 5 \%)$ \\
& Positive: $13 / 16(81 \cdot 3 \%) \dagger$ \\
& Negative: $3 / 16(18 \cdot 7 \%) \%$ \\
\hline
\end{tabular}

* The three smears with negative acid-fast bacilli (AFB) had a positive culture result.

$\uparrow$ Thirteen positive cultures for $M$. tuberculosis complex.

$\$$ Despite negative culture, the three were positive for AFB.

$\S$ Two patients did not present sufficient secretion and 14 did not collect it.

The T-SPOT.TB was positive in $4 / 25$ children $(16 \%)$ who had negative TST at the first evaluation; therefore, T-SPOT.TB added LTBI diagnosis to $16 \%$ (95\% CI 1.6-30-4) of cases. The QFT-IT was positive in $3 / 25$ children with negative TST, therefore it added LTBI diagnosis to $12 \%(95 \%$ CI $0 \cdot 0-24 \cdot 7)$ of cases.

When both tests were associated with TST, they added four diagnoses of LTBI for the 25 children with negative TST or $16 \%(95 \%$ CI $1 \cdot 6-30 \cdot 4)$.

Considering 24 children with negative T-SPOT.TB, there were two positive QFT-IT $(8 \cdot 3 \%, 95 \%$ CI $0 \cdot 0$ $19 \cdot 4)$; in the 24 negative QFT-IT, two were positive T-SPOT.TB $(8 \cdot 3 \%, 95 \%$ CI $0 \cdot 0-19 \cdot 4)$.

A multivariate logistic regression analysis was performed to assess the simultaneous influence of period of exposure and sleeping in the same room as index case upon the diagnosis of LTBI. Age $(P=0.688)$ and gender $(P=0.561)$ were not included in the model because they were not associated with LTBI in the univariate analysis.

The interaction between period of exposure and sleeping in the same room as index case was initially investigated and proved not to be significant $(P=$ $0 \cdot 839)$. It was therefore excluded from the model.

In the adjusted model, both the period of exposure $(P<0.001)$ and sleeping in the same room as index case $(P=0.024)$ were significant. Hence, the chance of LTBI in children and adolescents with a period of exposure of $\leqslant 60$ days was 6.9 times $(95 \%$ CI 1.9-26.0) higher than for those without known exposure to TB. For those with period of exposure $>60$ days, the chances of LTBI were 27 times higher (95\% CI 5.5-131·1). Moreover, the chances of LTBI in those who slept in the same room as an index case were $5 \cdot 2$ times $(95 \%$ CI $1 \cdot 2-22 \cdot 1)$ higher than for those who did not sleep in the same room (Table 4, Fig. 3).

Regarding the evolution of the test after treatment, TST had a significant incremental value of $1.8 \mathrm{~mm}$ $(P=0 \cdot 0021,95 \%$ CI $0 \cdot 289-3 \cdot 310)$ in the 40 children who were assessed after treatment. Only one adolescent was negative and became positive after the treatment. However, he had already been diagnosed by the other two tests.

The IGRAs were evaluated in 42 children after treatment, and no significant qualitative changes $(P>0.999)$ were observed for QFT-IT or T-SPOT.TB $(P=0.724)$ results. For T-SPOT.TB, there was a significant median reduction of 3.5 spots $(P=0.010)$ in panel A (ESAT-6 antigen) with a variation of -123 to +74 , and a median reduction of 3 spots $(P=0.035)$ in panel B (CFP-10 antigen), with a variation of -143 to +16 .

The concomitant use of three tests allowed the LTBI diagnosis in $41 / 59(69 \cdot 5 \%)$ children exposed to 
Table 3. Results of three tests of the the known-exposure and control groups at study entry and 24 weeks after treatment. Three patients (nos. 6, 12, 18) were retested after 8 weeks because they were negative at study entry and became positive on this occasion

\begin{tabular}{|c|c|c|c|c|c|c|c|c|c|c|}
\hline & \multicolumn{3}{|c|}{ Study entry } & \multicolumn{3}{|c|}{8 weeks after study entry } & \multicolumn{3}{|c|}{24 weeks after treatment } & \multirow[b]{2}{*}{ Abandon } \\
\hline & TST & T-SPOT.TB & QFT- IT & TST & T-SPOT.TB & QFT- IT & TST & T-SPOT.TB & QFT- IT & \\
\hline \multicolumn{11}{|c|}{ LTBI } \\
\hline 1 & $\mathrm{P}$ & I & $\mathrm{N}$ & & & & $\mathrm{N}$ & $\mathrm{N}$ & $\mathrm{P}$ & \\
\hline 2 & $\mathrm{P}$ & $\mathrm{P}$ & $\mathrm{P}$ & & & & $\mathrm{P}$ & $\mathrm{P}$ & $\mathrm{P}$ & \\
\hline 3 & $\mathrm{P}$ & I & $\mathrm{P}$ & & & & $\mathrm{P}$ & $\mathrm{N}$ & $\mathrm{N}$ & \\
\hline 4 & $\mathrm{P}$ & $\mathrm{N}$ & $\mathrm{P}$ & & & & $\mathrm{P}$ & $\mathrm{P}$ & $\mathrm{P}$ & \\
\hline 5 & $\mathrm{P}$ & $\mathrm{P}$ & $\mathrm{P}$ & & & & $\mathrm{P}$ & $\mathrm{P}$ & $\mathrm{P}$ & \\
\hline 6 & $\mathrm{~N}$ & $\mathrm{~N}$ & $\mathrm{~N}$ & $\mathrm{P}$ & $\mathrm{N}$ & $\mathrm{N}$ & $\mathrm{P}$ & $\mathrm{N}$ & $\mathrm{N}$ & \\
\hline 7 & $\mathrm{P}$ & $\mathrm{P}$ & $\mathrm{P}$ & & & & $\mathrm{P}$ & $\mathrm{P}$ & $\mathrm{P}$ & \\
\hline 8 & $\mathrm{P}$ & $\mathrm{P}$ & $\mathrm{P}$ & & & & $\mathrm{P}$ & $\mathrm{N}$ & $\mathrm{N}$ & \\
\hline 9 & $\mathrm{P}$ & $\mathrm{N}$ & $\mathrm{P}$ & & & & $\mathrm{P}$ & $\mathrm{P}$ & $\mathrm{P}$ & \\
\hline 10 & $\mathrm{~N}$ & $\mathrm{P}$ & $\mathrm{P}$ & & & & $\mathrm{N}$ & $\mathrm{N}$ & $\mathrm{P}$ & \\
\hline 11 & $\mathrm{P}$ & $\mathrm{P}$ & $\mathrm{P}$ & & & & $\mathrm{P}$ & $\mathrm{P}$ & $\mathrm{P}$ & \\
\hline 12 & $\mathrm{~N}$ & $\mathrm{~N}$ & $\mathrm{~N}$ & $\mathrm{P}$ & $\mathrm{P}$ & $\mathrm{P}$ & $\mathrm{P}$ & $\mathrm{P}$ & $\mathrm{P}$ & \\
\hline 13 & $\mathrm{P}$ & $\mathrm{P}$ & $\mathrm{P}$ & & & & $\mathrm{P}$ & $\mathrm{P}$ & $\mathrm{P}$ & \\
\hline 14 & $\mathrm{P}$ & $\mathrm{P}$ & $\mathrm{P}$ & & & & $\mathrm{P}$ & $\mathrm{N}$ & $\mathrm{P}$ & \\
\hline 15 & $\mathrm{P}$ & $\mathrm{P}$ & $\mathrm{P}$ & & & & $\mathrm{P}$ & $\mathrm{P}$ & $\mathrm{P}$ & \\
\hline 16 & $\mathrm{P}$ & $\mathrm{P}$ & $\mathrm{P}$ & & & & $\mathrm{P}$ & $\mathrm{P}$ & $\mathrm{P}$ & \\
\hline 17 & $\mathrm{P}$ & $\mathrm{P}$ & $\mathrm{P}$ & & & & & & & 2nd month \\
\hline 18 & $\mathrm{~N}$ & $\mathrm{~N}$ & $\mathrm{~N}$ & $\mathrm{~N}$ & $\mathrm{P}$ & $\mathrm{N}$ & $\mathrm{N}$ & $\mathrm{P}$ & $\mathrm{N}$ & \\
\hline 19 & $\mathrm{~N}$ & $\mathrm{P}$ & $\mathrm{N}$ & & & & $\mathrm{N}$ & $\mathrm{P}$ & $\mathrm{N}$ & \\
\hline 20 & $\mathrm{~N}$ & $\mathrm{P}$ & $\mathrm{P}$ & & & & $\mathrm{P}$ & $\mathrm{P}$ & $\mathrm{P}$ & \\
\hline 21 & $\mathrm{P}$ & $\mathrm{P}$ & $\mathrm{P}$ & & & & $\mathrm{P}$ & $\mathrm{P}$ & $\mathrm{P}$ & \\
\hline 22 & $\mathrm{P}$ & $\mathrm{P}$ & $\mathrm{P}$ & & & & n.d. & $\mathrm{P}$ & $\mathrm{P}$ & \\
\hline 23 & $\mathrm{P}$ & $\mathrm{P}$ & $\mathrm{P}$ & & & & n.d. & $\mathrm{P}$ & $\mathrm{P}$ & \\
\hline 24 & $\mathrm{P}$ & $\mathrm{N}$ & $\mathrm{N}$ & & & & $\mathrm{P}$ & $\mathrm{N}$ & $\mathrm{N}$ & \\
\hline 25 & $\mathrm{P}$ & $\mathrm{P}$ & $\mathrm{P}$ & & & & $\mathrm{P}$ & $\mathrm{P}$ & $\mathrm{P}$ & \\
\hline 26 & $\mathrm{P}$ & $\mathrm{P}$ & $\mathrm{N}$ & & & & $\mathrm{P}$ & $\mathrm{P}$ & $\mathrm{P}$ & \\
\hline 27 & $\mathrm{P}$ & $\mathrm{P}$ & $\mathrm{P}$ & & & & $\mathrm{P}$ & $\mathrm{P}$ & $\mathrm{P}$ & \\
\hline 28 & $\mathrm{P}$ & $\mathrm{P}$ & $\mathrm{P}$ & & & & $\mathrm{P}$ & $\mathrm{P}$ & $\mathrm{P}$ & \\
\hline 29 & $\mathrm{P}$ & $\mathrm{P}$ & $\mathrm{P}$ & & & & $\mathrm{P}$ & $\mathrm{P}$ & $\mathrm{P}$ & \\
\hline 30 & $\mathrm{P}$ & $\mathrm{P}$ & $\mathrm{P}$ & & & & $\mathrm{P}$ & $\mathrm{P}$ & $\mathrm{P}$ & \\
\hline 31 & $\mathrm{P}$ & $\mathrm{P}$ & $\mathrm{P}$ & & & & $\mathrm{P}$ & $\mathrm{P}$ & $\mathrm{P}$ & \\
\hline 32 & $\mathrm{P}$ & $\mathrm{P}$ & $\mathrm{P}$ & & & & $\mathrm{P}$ & $\mathrm{P}$ & $\mathrm{P}$ & \\
\hline 33 & $\mathrm{P}$ & $\mathrm{P}$ & $\mathrm{P}$ & & & & & & & 5th month* \\
\hline 34 & $\mathrm{P}$ & $\mathrm{P}$ & $\mathrm{P}$ & & & & & & & 5th month* \\
\hline 35 & $\mathrm{P}$ & $\mathrm{P}$ & $\mathrm{P}$ & & & & $\mathrm{P}$ & $\mathrm{P}$ & $\mathrm{P}$ & \\
\hline 36 & $\mathrm{~N}$ & $\mathrm{P}$ & $\mathrm{P}$ & & & & $\mathrm{N}$ & $\mathrm{P}$ & $\mathrm{P}$ & \\
\hline 37 & $\mathrm{P}$ & $\mathrm{P}$ & $\mathrm{P}$ & & & & $\mathrm{P}$ & $\mathrm{P}$ & $\mathrm{P}$ & \\
\hline 38 & $\mathrm{P}$ & $\mathrm{P}$ & $\mathrm{P}$ & & & & $\mathrm{P}$ & $\mathrm{P}$ & $\mathrm{P}$ & \\
\hline 39 & $\mathrm{P}$ & $\mathrm{P}$ & $\mathrm{P}$ & & & & $\mathrm{P}$ & $\mathrm{P}$ & $\mathrm{P}$ & \\
\hline 40 & $\mathrm{P}$ & $\mathrm{P}$ & $\mathrm{P}$ & & & & $\mathrm{P}$ & $\mathrm{N}$ & $\mathrm{N}$ & \\
\hline 41 & $\mathrm{P}$ & $\mathrm{P}$ & $\mathrm{P}$ & & & & $\mathrm{P}$ & $\mathrm{P}$ & $\mathrm{P}$ & \\
\hline \multicolumn{11}{|c|}{ Control group } \\
\hline 42 & $\mathrm{P}$ & $\mathrm{P}$ & $\mathrm{P}$ & & & & $\mathrm{P}$ & $\mathrm{P}$ & $\mathrm{P}$ & \\
\hline 43 & $\mathrm{P}$ & $\mathrm{N}$ & $\mathrm{N}$ & & & & $\mathrm{N}$ & $\mathrm{N}$ & $\mathrm{N}$ & \\
\hline 44 & $\mathrm{~N}$ & $\mathrm{~N}$ & $\mathrm{P}$ & & & & $\mathrm{N}$ & $\mathrm{N}$ & $\mathrm{N}$ & \\
\hline 45 & $\mathrm{P}$ & $\mathrm{N}$ & $\mathrm{N}$ & & & & $\mathrm{P}$ & $\mathrm{N}$ & $\mathrm{N}$ & \\
\hline
\end{tabular}

P, Positive; N, negative; I, indeterminate; n.d., not done.

* Abandoned follow-up in the 5th month, but came back for clinical evaluation 2 months after and were both well. 
(a)

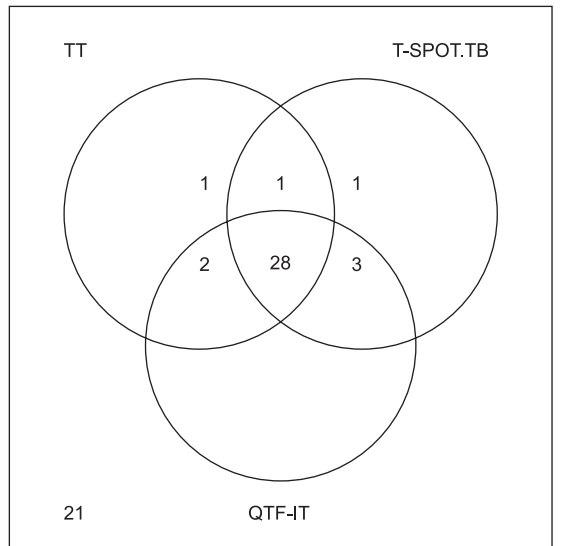

(b)

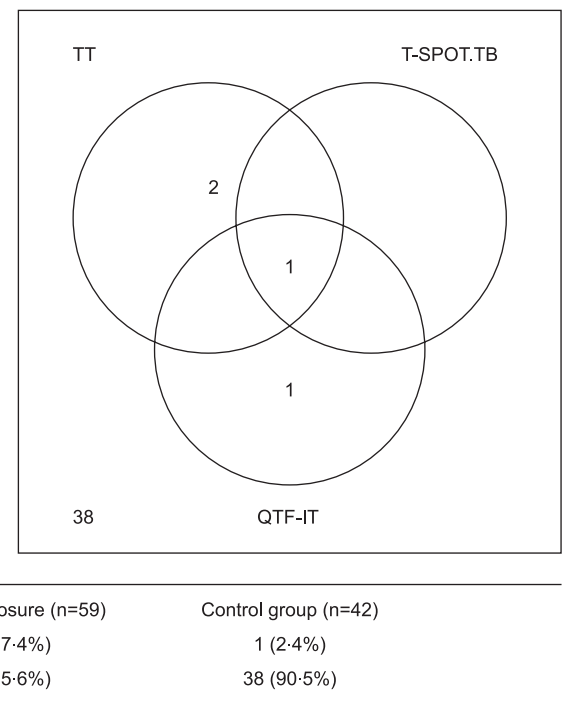

$$
\begin{array}{ccc}
\text { Results of three tests } & \text { Known exposure }(n=59) & \text { Control group }(n=42) \\
\text { All positive } & 28(47 \cdot 4 \%) & 1(2 \cdot 4 \%) \\
\text { All negative } & 21(35 \cdot 6 \%) & 38(90.5 \%)
\end{array}
$$

Kappa - TT x QFT-IT= 0.807; TT x T-SPOT.TB= 0.798; QFT-IT x T-SPOT.TB= 0.888

Fig. 2. Agreement and disagreement between the three tests (TST, QFT-IT, T-SPOT.TB) in (a) the known exposure group and $(b)$ the control group at the first evaluation.

active TB and in 4/42 (9.5\%) children without known contact with a TB patient.

In the known exposure group, if only TST was employed, LTBI would be diagnosed in $61 \%$ (95\% CI 48.6-73.5) of cases; if only QFT- IT was employed, $59 \cdot 3 \%$ (95\% CI 46.8-71.9) of LTBI cases would be diagnosed. If only T-SPOT.TB was used, LTBI would be diagnosed in $57 \cdot 6 \%(95 \%$ CI $45 \cdot 0-70 \cdot 2)$ of cases.

\section{DISCUSSION}

This is a prospective study conducted exclusively on children and adolescents who presented a high rate of LTBI in those exposed to an adult with active pulmonary TB. No child progressed to active disease during the follow-up. There was a high agreement in results obtained with TST and IGRAs. However, an increment in LTBI diagnosis was observed either with TST repetition or with the association of IGRAs to TST. After treatment, no significant change was observed in the results of the three tests.

The infection rate of $69 \cdot 5 \%$ was higher than what has been reported by other Brazilian studies that evaluated children by TST, with rates between $30 \%$ and $58 \%$ [2022]. Today, Brazil has a moderate prevalence of TB with an incidence rate of $36 \cdot 1 / 100000$ concentrated in some risk groups such as prisoners, Native Indians, homeless people and HIV-infected individuals [23]. However, this study was performed in the city of São Paulo, which has a high TB coefficient incidence (46.9/100 000) [24]. That is probably due to the presence of urban areas that concentrate a high proportion of TB patients who live in conditions of extreme poverty and substandard housing that facilitate the transmission of the disease. Therefore it is very important that LTBI in children and adolescents is diagnosed and treated to avoid progression to TB disease [3].

Studies that evaluated the risk of TB infection in countries with high incidence of the disease using other tests combined with TST detected rates of infection in contacts from $24 \%$ to $74 \%[8,9,25]$.

The risk of an exposed individual becoming infected is related to the infectiousness of the index case [20], duration and proximity of contact $[13,20]$ and the susceptibility of the exposed person [26].

The assessment of the infection rate is determined by the way the diagnostic investigation is undertaken, i.e. which test is selected [20, 22].

The diagnostic value of TST depends on the cut-off value used to distinguish positive from negative results [18], on the use of BCG vaccine in the population analysed, on the cross-reactivity with non-tuberculous environmental mycobacteria (NTM), on nutritional status and on immunosuppressive conditions present at contact [27]. 
Table 4. Logistic regression analyses for the assessment of demographic and clinical characteristics associated with latent tuberculosis infection (LTBI) diagnosis

\begin{tabular}{|c|c|c|c|}
\hline Parameters & $\mathrm{LTBI}+(n=45)$ & LTBI- $(n=56)$ & $P$ value \\
\hline \multicolumn{4}{|l|}{ Univariate analysis } \\
\hline Gender & & & 0.561 \\
\hline Male & 23 & 25 & \\
\hline Female & 22 & 31 & \\
\hline Age & & & $0 \cdot 688$ \\
\hline$\leqslant 5$ years & 17 & 19 & \\
\hline$>$ 5years & 28 & 37 & \\
\hline Length of exposure & & & $<0 \cdot 001$ \\
\hline No exposure & 4 & 38 & \\
\hline $1-60$ days & 16 & 14 & \\
\hline$>60$ days & 25 & 4 & \\
\hline \multicolumn{4}{|l|}{ Sleeping } \\
\hline In the same room & 24 & 3 & $<0 \cdot 001$ \\
\hline In the same house & 41 & 18 & \\
\hline No exposure & 4 & 38 & \\
\hline Parameters & OR & $(95 \% \mathrm{CI})$ & \\
\hline \multicolumn{4}{|l|}{ Multivariate analysis } \\
\hline No TB contact $(n=42)$ & - & - & \\
\hline $1-60$ days of TB contact $(n=30)$ & $6 \cdot 9$ & $(1 \cdot 85-26 \cdot 03)$ & \\
\hline$>60$ days of TB contact $(n=29)$ & $27 \cdot 0$ & $(5 \cdot 55-131 \cdot 08)$ & \\
\hline Sleeping in the same room $(n=27)$ & $5 \cdot 2$ & $(1 \cdot 24-22 \cdot 07)$ & \\
\hline
\end{tabular}

OR, Odds ratio; CI, confidence interval.

The recent reduction of the TST cut-off value in Brazil increased test sensitivity [28] and allowed the diagnosis of six children in the present study, who would not be considered infected if the previous cutoff was used. All these six children also had positive IGRAs (QFT-IT and T-SPOT.TB), suggesting that they were indeed infected.

However, the exclusive use of the TST would prevent the diagnosis of TB in a 3-month-old infant who had a TST result of $5 \mathrm{~mm}$ but who was treated because the two IGRAs turned positive.

In Brazil, the repetition of TST in individuals exposed to active TB is recommended after 6-12 weeks when the first result is negative [18]. In the present study, this procedure detected two cases that would otherwise have been considered negative, showing the importance of this recommendation.

Despite the known difficulties inherent to the TST - low sensitivity, low specificity, the possibility of errors in the application and reading and the need to return in $72 \mathrm{~h}$ to read the result - the infection rate detected exclusively by this test in this study was also higher $(61 \%$ for the treatment group and $7 \cdot 1 \%$ for the control group) than what has been described in the literature [20-22].

BCG vaccine administered in the neonatal period has been shown to have minimal impact on the specificity of TST. In Spain, researchers also noted that BCG given at birth does not influence the outcome of TST 3 years after its administration [29].

Regarding the presence of NTM interfering with the specificity of TST, the State of São Paulo has a low number of cases reported compared to other regions in Brazil [30]. Therefore, it is unlikely that these infections have affected the response of TST in the present study.

Some studies published over the last decade using IGRAs showed that BCG given in childhood might protect against $M$. tuberculosis infection [31, 32]. In the present study, $98.3 \%$ of children had been vaccinated with BCG at birth; however, despite that, a high rate of LTBI was observed.

No child developed active disease in our study. It is known that malnutrition is one of the factors that contributes to the development of TB disease [33]. Perhaps the good nutritional status of children and adolescents in 
Fisher's Exact Test

$P<0.001$
$94 \cdot 5 \%$

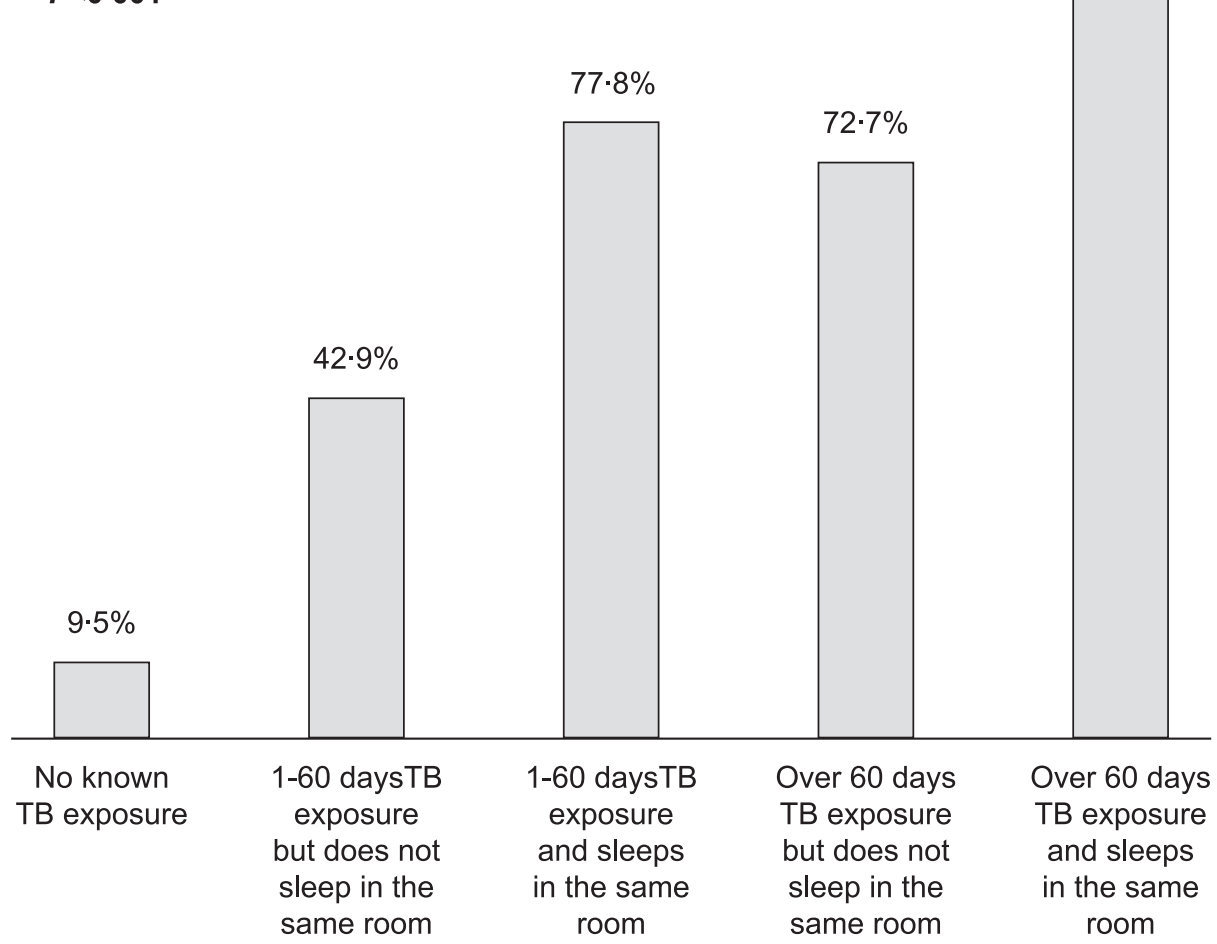

Fig. 3. Latent tuberculosis infection rate in children and adolescents according to the exposure period and sleeping in the same room.

our sample has contributed to the absence of TB disease: only four children were malnourished and still had a borderline body mass index $Z$ score of $-2 \cdot 02$ to $-2 \cdot 21$.

In the absence of a gold standard for the diagnosis of LTBI, interpreting discordant results is a challenge. Factors associated with discordance between tests are the age of the exposed child [14] the presence of BCG (age of administration and number of doses) [29] as well as the necessary period of time required by different tests to turn positive [34].

In general, in areas of low and moderate prevalence of TB, the agreement between the tests depends on the vaccination status of the participants, being higher in the unvaccinated population and lower in those BCG vaccinated [35].

The high agreement between tests observed in this study in a population so widely vaccinated suggests that a single dose of BCG at birth does not reduce the specificity of TST [29].

Another factor that may have contributed to the high agreement found could be the prolonged exposure to the index case $->30$ days in $74.5 \%$ of the sample - probably enough for both TST and IGRAs to turn positive [34].
Despite this high agreement, the use of IGRAs contributed to the diagnosis in some cases. The literature suggests that the consecutive use of tests is possible when one desires to increase their sensitivity [13, 25].

On the other hand, only the repetition of TST in 6-12 weeks led to a diagnosis increment. There are no data on the use of this strategy in other countries.

Importantly, the repetition of TST should not be confused with the 'PPD booster' strategy [18].

It is worth mentioning the possible effect of TST on subsequent IGRA results. In previous studies, different IGRAs have been tested and varied periods of time between the two tests have been evaluated, providing some evidence that TST can boost a subsequent IGRA result; however, this is more apparent when the IGRA is positive to begin with and when the second IGRA is performed 7-28 days after the TST [36].

The fact that our results have shown that the three tests (TST, QFT-IT, T-SPOT.TB) have a similar performance in Brazilian children exposed to adults with active pulmonary TB is encouraging. Since 2013 Brazil has been facing a shortage of PPD. However, both QFT-IT and T-SPOT.TB are still quite expensive to use as a substitute for TST in the routine of TB 
diagnosis for immunocompetent individuals and are not usually available in Brazil [18]. The introduction of IGRAs in Brazil might take place in the future, at least initially for immunosuppressed patients. Fortunately, PPD has recently been purchased by the Ministry of Health again and it is gradually being distributed to healthcare units in Brazil.

There is limited data on the use of IGRAs in some individuals, such as healthcare workers who are repeatedly tested and children aged $<5$ years [37]. In our study, 26/ $59(44 \%)$ of TB-exposed children were aged $<5$ years (range 0.3-4.6 years). Interestingly, in this subgroup, the agreement between the three tests was also high.

In line with the literature $[9,25,35,38]$, logistic regression analysis showed that children with known exposure to an adult with active pulmonary $\mathrm{TB}$ were 6.9-27 times more likely to be infected than those with no known contact with TB. Sleeping in the same room as an adult with active pulmonary $\mathrm{TB}$ would increase this risk $5 \cdot 2$ times further.

Test results were not modified by treatment, suggesting that there is no advantage in their repetition. However, a quantitative variation after treatment was observed both after TST - with a significant increase in response - and after T. SPOT.TB, where there was a significant decrease in the number of IFN- $\gamma$-producing cells when stimulated with specific antigens for $M$. tuberculosis.

A previous study using Quantiferon did not show differences pre- and post-treatment in children treated for LTBI or in the group treated for TB disease [39].

A decrease in the number IFN- $\gamma$-producing cells in T.SPOT.TB does not necessarily indicate a change in immune response because other cytokines involved were not measured.

This study is limited by its small sample size, as reflected in the wide confidence intervals around effect estimates despite statistically significant results for some outcomes. Another limitation was the inability to collect sputum for mycobacteria culture from all index cases.

On the other hand, the high adherence to the proto$\mathrm{col}$ - a product of the follow-up of children performed by the same professional, of the attention given to the family in the health unit and of the reimbursement of the cost of transportation to all appointments allowed the vast majority to pursue all proposed steps of the study. These factors have already been reported previously in Brazil [40] and also in Tanzania [41].

\section{CONCLUSION}

In our setting, in immunocompetent children and adolescents exposed to an adult with active pulmonary TB, TST had a similar performance to IGRAs and detected a high rate of LTBI.

\section{ACKNOWLEDGEMENTS}

This study was funded by Fundação de Amparo à Pesquisa do Estado de São Paulo (FAPESP), Brazil: 2009/07603-7.

\section{DECLARATION OF INTEREST}

None.

\section{REFERENCES}

1. Marais BJ, et al. The natural history of childhood intra-thoracic tuberculosis: a critical review of literature from the pre-chemotherapy era. International Journal of Tuberculosis and Lung Disease 2004; 8: 392-402.

2. Singh M, et al. Latent tuberculosis in children: diagnosis and management. Indian Journal of Pediatrics 2011; 78: 464-468.

3. Health Ministry of Brazil (MSB). Secretariat of Health Surveillance. Epidemiological Surveillance Department. Guidelines for tuberculosis control in Brazil. Health Ministry of Brasil, 2011, 284 pp.

4. Lalvani A, Pareek M. A 100 year update on diagnosis of tuberculosis infection. British Medical Bulletin 2010; 93: 69-84.

5. Lalvani A, et al. Rapid detection of Mycobacterium tuberculosis infection by enumeration of antigen-specific $\mathrm{T}$ cells. American Journal of Respiratory and Critical Care Medicine 2001; 163: 824-828.

6. Ferrara G, et al. Use in routine clinical practice of two commercial blood tests for diagnosis of infection with Mycobacterium tuberculosis: a prospective study. Lancet 2006; 367: 1328-1334.

7. Hesseling AC, et al. Highly discordant $\mathrm{T}$ cell responses in individuals with recent exposure to household tuberculosis. Thorax 2009; 64: 840-846.

8. Nakaoka H, et al. Risk for tuberculosis among children. Emerging Infectious Diseases 2006; 12: 1383-1388.

9. Okada K, et al. Performance of an interferon-gamma release assay for diagnosing latent tuberculosis infection in children. Epidemiology and Infection 2008; 136: 1179-1187.

10. Cruz AT, et al. Comparing the tuberculin skin test and T-SPOT.TB blood test in children. Pediatrics 2011; 127: e31-38.

11. Altet-Gómez N, et al. Diagnosing TB infection in children: analysis of discordances using in vitro tests and the tuberculin skin test. European Respiratory Journal 2011; 37: 1166-1174. 
12. Critselis E, et al. The effect of age on whole blood interferon-gamma release assay response among children investigated for latent tuberculosis infection. Journal of Pediatrics 2012; 161: 632-638.

13. Yassin MA, et al. Use of tuberculin skin test, IFN- $\gamma$ release assays and IFN- $\gamma$-induced protein-10 to identify children with TB infection. European Respiratory Journal 2013; 41: 644-648.

14. Blandinières A, et al. QuantiFERON to diagnose infection by Mycobacterium tuberculosis: performance in infants and older children. Journal of Infection 2013; 67: 391-398.

15. World Health Organization. WHO Anthro. Version 3.2.2 (www.who.int/childgrowth/software/en/). Geneva: World Health Organization, 2011.

16. World Health Organization. WHO Anthro Plus (www. who.int/growthref/tools/en/). Geneva: World Health Organization, 2007.

17. ABEP. Brazilian association of Research Companies Socio Economic Classification Criterion Brazil (CCEB) 2009 (http://www.abep.org/new/criterioBrasil. aspx). Accessed January 2009.

18. Conde MB, et al. III Brazilian Thoracic Association Guidelines on tuberculosis. Jornal Brasileiro de Pneumologia 2009; 35: 1018-1048.

19. Pedrozo C, et al. Efficacy of the scoring system, recommended by the Brazilian National Ministry of Health, for the diagnosis of pulmonary tuberculosis in children and adolescents, regardless of their HIV status. Jornal Brasileiro de Pneumologia 2010; 36: 92-98.

20. Almeida LM, et al. Use of purified protein derivative to acess the risk of infection in children in close contact with adults with tuberculosis in a population with high Calmette-Guerrin bacillus coverage. Pediatric Infectious Diseases Journal 2001; 20: 1061-1065.

21. Caldeira ZMR, Sant'Anna CC, Aidé MA. Tuberculosis contact tracing among children and adolescents, Brazil. Revista de Saude Publica 2004; 38: 339-345.

22. Maciel EL, et al. Juvenile household contacts aged 15 or younger of patients with pulmonary TB in the greater metropolitan area of Vitória, Brazil: a cohort study. Jornal Brasileiro de Pneumologia 2009; 35: 359-366.

23. Health Ministry of Brazil (MSB). Tuberculosis: aligned with the social, in tune with the technology. Boletim Epidemiológico 2013; 44: 1-6.

24. Health Ministry of Brazil (MSB). Tuberculosis control in Brazil: advances, innovations and challenges. Boletim Epidemiológico 2014; 44: 1-13.

25. Adetifa IM, et al. Commercial interferon gamma release assays compared to the tuberculin skin test for diagnosis of latent Mycobacterium tuberculosis infection in childhood contacts in the Gambia. Pediatric Infectious Diseases Journal 2010; 29: 439-443.
26. Dheda K, et al. The immunology of tuberculosis: from bench to bedside. Respirology 2010; 15: 433-450.

27. Whitworth HS, et al. IGRAs - the gateway to T cell based TB diagnosis. Pediatric Pulmonology 2013; 61: 52-62.

28. Cailleaux-Cezar M, et al. Tuberculosis incidence among contacts of active pulmonary tuberculosis. International Journal of Tuberculosis and Lung Disease 2009; 13: 190-195.

29. Piñeiro R, et al. Tuberculin skin test in bacille Calmette-Guérin-vaccinated chidren: how should we interpret the results? European Journal of Pediatrics 2012; 171: $1625-1632$.

30. Wildner LM, Nogueira CL, Souza S. Mycobacteria: epidemiology and diagnosis. Revista de Patologia Tropical 2011; 40: 207-229.

31. Eriksen $\mathbf{J}$, et al. Protective effect of BCG vaccination in a nursery outbreak in 2009: time to reconsider the vaccination threshold? Thorax 2010; 65: 1067-1071.

32. Basu R, et al. Identifying predictors of interferon- $\gamma$ release assay results in pediatric latent tuberculosis: a protective role of bacillus Calmette-Guérin? A pTBNET Collaborative Study. American Journal Respiratory and Critical Care Medicine 2012; 186: 378-384.

33. Guimarães RM, et al. Tuberculosis, HIV and poverty: time trend in Brazil, the Americas and the world. Jornal Brasileiro de Pneumologia 2012; 38: 511-517.

34. Lee SW, et al. Time interval to conversion of interferon- $\gamma$ release assay after exposure to tuberculosis. European Respiratory Journal 2011; 37: 1447-1452.

35. Méndez-Echevarría A, et al. Interferon- $\gamma$ release assay for the diagnosis of tuberculosis in children. Archives of Disease in Childhood 2012; 97: 514-516.

36. van Zyl-Smit RN, et al. Within-subject variability of interferon-g assay results for tuberculosis and boosting effect of tuberculin skin testing: a systematic review. PLoS ONE 2009; 4: e8517.

37. Centers for Disease Control and Prevention. Updated guidelines for using interferon gamma release assay to detect Mycobacterium tuberculosis infection-United States, 2010. Morbidity and Mortality Weekly Report 2010; 59: 1-25.

38. Rutherford ME, et al. QuantiFERON ${ }^{\circledR}-\mathrm{TB}$ Gold In-Tube assay vs. tuberculin skin test in Indonesian children living with a tuberculosis case. International Journal of Tuberculosis and Lung Disease 2012; 16: 496-502.

39. Nenadić $\mathbf{N}$, et al. Serial interferon- $\gamma$ release assay in children with latent tuberculosis infection and children with tuberculosis. Pediatric Pulmonology 2012; 47: 401-408.

40. Machado Jr. A, et al. Analysis of discordance between the tuberculin skin test and the interferon-gamma release assay. International Journal of Tuberculosis and Lung Disease 2009; 13: 446-453.

41. Nissen TN, et al. Challenges of loss to follow-up in tuberculosis research. PLOS ONE 2012; 7: e40183. 